\title{
Validación y fiabilidad del dispositivo Haefni Health System 1.0 en la medición de la velocidad en el rango isocinético
}

\section{Validity and Reliability of the Haefni Health System 1.0 device in the measurement of the isokinetic velocity range}

\section{Validação e fidelidade do dispositivo Haefni Health HHe 1.0 na medição da velocidade e da amplitude isocinética}

\author{
Christian Alex Campos Jara ${ }^{1}$, Iker Javier Bautista González ${ }^{2}$, Luis Javier Chirosa Ríos ${ }^{2}$, \\ Ignacio Martin Tamayo ${ }^{1,2}$, Antonio Eduardo Lopez Fuenzalida e Ignacio Jesús Chirosa Ríos ${ }^{2}$ \\ 1 Pontificia Universidad Católica de Chile. Santiago, Chile; 2 Universidad de Granada. España.
}

\begin{abstract}
Resumen: Cuando se realizan valoraciones de cualquier protocolo de evaluación uno de los aspectos fundamentales es conocer la validez y fiabilidad de los dispositivos utilizados. El objetivo del presente trabajo fue analizar la validez y fiabilidad de la variable de velocidad en el rango isocinético (VRI) en el dispositivo Haefni Health (HHe 1.0). Un total de dos protocolo se diseñaron para el análisis de la validez y fiabilidad. Los resultados mostraron altos Índices de Correlación Intraclase $\left(\mathrm{ICC}_{2,1}\right)$ para las medidas de validez y fiabilidad del dispositivo $(0,998$ y 0,99 , respectivamente). Cuando se analizó la fiabilidad de las medidas por cada una de las condiciones de evaluación altos ICC $_{2,1}$, además de bajos CV y SEM fueron encontraros (rango $0,71-0,99$, rango $0,19-3,73$ y rango $0,001-0,004 \mathrm{~m} \cdot \mathrm{s}^{-1}$, respectivamente). Los resultados obtenidos avalan la validez y fiabilidad del dispositivo Haefni Health HHe1.0 para la medición de la variable de VRI tanto para la fase concéntrica como para la fase excéntrica del movimiento.

Palabras clave: precisión, validación, fiabilidad, velocidad, dispositivo isocinético

Abstract: When rating any assessment protocol one of the key issues is to determine the validity and reliability of the devices used. The aim of this study was to analyze the validity and reliability of the variable velocity isokinetic range (VRI) in Haefni Health System (HHe 1.0). Two assessment protocols were design to test bot, validity and reliability. The results showed high Index of Intraclass Correlation ( $\mathrm{ICC}_{2,1}$ ) for measures of validity and
\end{abstract}

reliability of the VRI $(0,998$ and 0,99 , respectively). Moreover, when the reliability was assessed at each criterion velocity high values of $\mathrm{ICC}_{2,1}$, range $0,71-0,99$ and low CV and SEM, range $0,19-3,73 \%$ and range $0.001-$ $0.004 \mathrm{~m} \cdot \mathrm{s}^{-1}$, were found respectively. The results obtained confirm the validity and reliability of HHe1.0 device for measuring VRI variable.

Keywords: accuracy, validation, reliability, velocity, isokinetic device

Resumo: Quando se realizam apreciaçôes de qualquer protocolo de avaliaçáo um dos aspectos fundamentais é conhecer a validade e fidelidade dos dispositivos utilizados. O objectivo do presente trabalho foi analisar a validade e fidelidade da variável de velocidade na amplitude isocinética (VRI) no dispositivo Haefni Health HHe 1.0. Um total de dois protocolos foram delineados para a análise da validade e fidelidade. Os resultados mostraram elevados Índices de Correlação Intraclasse $\left(\mathrm{ICC}_{2,1}\right)$ para as medidas de validade e fidelidade do dispositivo (0,998 e 0,99, respectivamente). Adicionalmente, quando se analisou a fidelidade das medidas por critério de altos ICC ${ }_{2,1}$ e baixos CV e SEM foram identificados (amplitude 0,71-0,99, amplitude $0,19-3,73$ e amplitude $0,001-0,004 \mathrm{~m} \cdot \mathrm{s}^{-1}$, respectivamente). Os resultados obtidos confirmam a validade e fidelidade do dispositivo Haefni Health HHe1.0 para a medição da variável de VRI, tanto para a fase concêntrica, como para a fase excêntrica do movimento.

Palavras-chave: precisão, validaçấo, fidelidade, velocidade, dispositivo isocinético.

\section{Introducción}

Cuando se realiza cualquier tipo de intervención es conveniente seleccionar aquellos protocolos de evaluación que sean válidos y fiables para un determinado fin. Del mismo modo, se deberán seleccionar aquellos dispositivos de evaluación válidos y fiables para la medición de una determinada variable. Las fuentes de error dentro del proceso de evaluación son atribuibles principal-

\footnotetext{
Dirección para correspondencia [Correspodence address]: Christian Alex Campos Jara, Physical Therapy School, Faculty of Medicine, Pontificia Universidad Católica de Chile, Av. Vicuńa Mackenna 4860, zip code 7820436, Macul, Santiago, Chile. E-mail: ccamposj@uc.cl. Telephone +56223541307 .
}

mente a las diferencias biológicas de los participantes. Sin embargo, otra fuente de variación puede ser el propio instrumento utilizado para la obtención de los datos (Hopkins, 2000; Weir, 2005). Partiendo de este supuesto, todo dispositivo de evaluación debe tener dos condiciones fundamentales, que sean válidos y fiables, con el fin de poder detectar diferencias o cambios entre los diferentes sujetos evaluados. En este sentido, el concepto de validez se entiende como la capacidad del dispositivo para medir aquello que pretendemos medir. Mientras que cuando se habla de fiabilidad se concibe como la estabilidad en la medida tras múltiples repeticiones. En otras palabras, la ausencia de error de la 
medida (Atkinson, y Neville, 1998). Aunque siendo realistas, en todo proceso de medición siempre existirá un componente error.

Generalmente, para analizar la validez y fiabilidad de un nuevo dispositivo de evaluación se utiliza lo que se conoce como un "gold estándar", es decir, otro dispositivo que mide con un escaso margen de error aquello que pretendemos evaluar. Posteriormente, los datos obtenidos en ambos dispositivos (los procedentes del gold estándar y las del nuevo dispositivo a testar) son comparadas y sometidas a tratamiento estadístico para comprobar su validez concurrente. En el caso específico de la fiabilidad, las pruebas test-retest son las más utilizadas para valorar la estabilidad en la medida tras múltiples repeticiones. En este sentido, existen muchos estadísticos para evaluar la fiabilidad. Uno de los más extendidos es el Índice de Correlación Intraclase (ICC) y el Error Estándar de la Medida (SEM). Ambos procedimientos, junto a otros tipos de indicadores estadísticos como el coeficiente de variación $(\mathrm{CV})$ o la media, la desviación estándar y el gráfico BlandAltman de las diferencias, son procedimientos estadísticos que ofrecen una idea clara de la fiabilidad de cada una de las medidas del dispositivo evaluado. Por lo tanto, según Atkinson y Neville (1998) sería conveniente presentar no sólo un indicador de la fiabilidad, si no todos ellos de forma conjunta, puesto que la interpretación de los resultados será mucho más clara. Otras recomendaciones que realizan estos autores son: (a) las medidas de fiabilidad de una muestra corresponde a dicha muestra, por lo tanto, siempre que se realice un nuevo estudio con cualquier dispositivo sería conveniente realizar nuevamente las medidas de fiabilidad. Esto es así porque la propia variabilidad biológica de la muestra utilizada en ese estudio en concreto puede estar influenciando en los datos obtenidos. (b) Hay que recordar que se considera una buena fiabilidad cocientes superiores a 0,90.

A lo largo de la bibliografía existente en el tema es posible encontrar numerosos estudios que analizan la validez y la fiabilidad de distintos dispositivos de evaluación. Requena, Requena, García, Saez-Saez de Villarreal, y Pääsuke (2012) analizaron la validez y la fiabilidad de un dispositivo (keimo$\mathrm{ve}^{\mathrm{TM}}$ ) para medir el salto vertical. Dichos autores utilizaron una plataforma de fuerzas, un dispositivo de desplazamiento lineal y una cámara de alta velocidad como "gold estándar" para la evaluación de las variables de fuerza, velocidad y altura del salto, respectivamente. La muestra utilizada para la evaluación del salto con contramovimiento fueron un total de 13 futbolistas profesionales. Los resultados mostraron una buena validez $(r=0,99, \mathrm{p}<0,01)$ entre las medidas del nuevo dispositivo comparadas con el gold estándar. Los ICC's y CV's de las variables de velocidad de despegue, altura de salto y fuerza estuvieron en un rango de 0,92-0,97 y 2,1-7,4, respectivamente. Aunque en este sentido, debemos de seńalar una diferencia importante cuando hablamos de los términos de "validez" y "fiabilidad" referido a los dispositivos de evaluación. Sin embargo, cuando se realizan pruebas con el objetivo principal de analizar la validez y fiabilidad de un dispositivo, no debería participar ninguna persona, puesto que esta misma está introduciendo una fuente de error importante (estandarización, motivación a la hora de realizar el test, técnica, instrucciones). En este sentido, en el error total encontrado no tendremos conocimiento si es procedente del dispositivo de evaluación o simplemente, de un mal proceso de estandarización. Este concepto a pesar de parecer sencillo, es posible encontrar investigaciones (Sole, Hamrén, Milosavljevic, Nicholson, y Sullivan, 2007; Toonstra y Mattacola, 2012; Tsiros, Grimshaw, Shield, y Buckley, 2011) que analizan la "validez" y "fiabilidad" de un dispositivo de evaluación pero usando personas para dichas pruebas. Por lo tanto, habrá que diferenciar entre la pruebas de "validez" y "fiabilidad" de un dispositivo de evaluación y las pruebas de "validez" y "fiabilidad" de un protocolo de evaluación de un ejercicio en concreto. En el primer caso estaríamos obteniendo una medida de validez y fiabilidad del dispositivo para la evaluación de las variables que deseemos testar. Mientras que en el segundo caso, se obtendrían unos datos para la evaluación de la validez y fiabilidad enmarcados dentro de un ejercicio en concreto. Es importante resaltar que ambas comprobaciones son igualmente de importantes. Más específicamente, Drouin, Valovich-mcLeod, Shultz, Gansneder, y Perrin (2004) analizaron la validez y fiabilidad mecánica del dispositivo isocinético Biodex System 3 pro en las variables de velocidad, posición y torque. Estos autores evaluaron tanto la fiabilidad en el día (trial-to-trial) y la fiabilidad entre días (day-to-day). Los autores concluyeron que dicho dispositivo era válido y fiable tanto para propósitos clínicos como de investigación. Sin embargo, cabe resaltar que también advirtieron que ningún estudio hasta la fecha había evaluado aun la fase excéntrica de dicho dinamómetro. Por su parte, Patterson y Spivey (1992) analizaron la validez y fiabilidad del dispositivo isocinético LIDO Active para las variables de la velocidad y el torque. La velocidad testada fue de $5 \%$ s con un ROM de $200^{\circ}$, tanto en la fase concéntrica como en la excéntrica. El análisis de la varianza no mostró diferencias significativas en ninguna de las medidas proporcionadas. La correlación de Pearson entre los valores de torque en ambos días de evaluación fue de $r$ $=1$. Desafortunadamente, en el mencionado estudio no se presentó ningún estadístico de ICC, SEM o CV.

Recientemente, se ha introducido en el mercado de la rehabilitación/actividad física y salud un nuevo dispositivo que permite tanto la evaluación como el entrenamiento-tratamiento que posee, entre otros, un modo de trabajo isocinético. A diferencia de otros dispositivos isocinéticos, este dispositivo (Haefni Health System 1.0; HHe 1.0) genera velocidades isocinéticas lineales. Por lo tanto, los objetivos de esta investigación fueron (a) analizar la validez de velocidad en el rango isocinético (VRI) y (b) analizar la fiabilidad de la VRI del dispositivo 
HHe 1.0. Todas estas pruebas se realizaron tanto en la fase concéntrica como en la excéntrica del movimiento.

\section{Método}

\section{Procedimiento}

Todas las pruebas fueron realizadas en el laboratorio de la empresa iVolution RyD (Granada, España).

Para la medición de la velocidad, el cable del dispositivo de desplazamiento lineal (DDL) fue enganchado al extremo proximal del sistema HHe 1.0 (ver Figura 1). De este modo, en el momento de ejecutar las repeticiones se ponía en marcha el DDL registrando la variable de la velocidad en todo el ROM.

Figura 1. Representación esquemática del dispositivo de desplazamiento lineal enganchado al extremo distal del dispositivo HHe 1.0. La línea negra continua representa la fase de ida (concéntrica) y la línea discontinua la fase de vuelta (excéntrica). El recuadro gris representa todo el rango de movimiento evaluado.

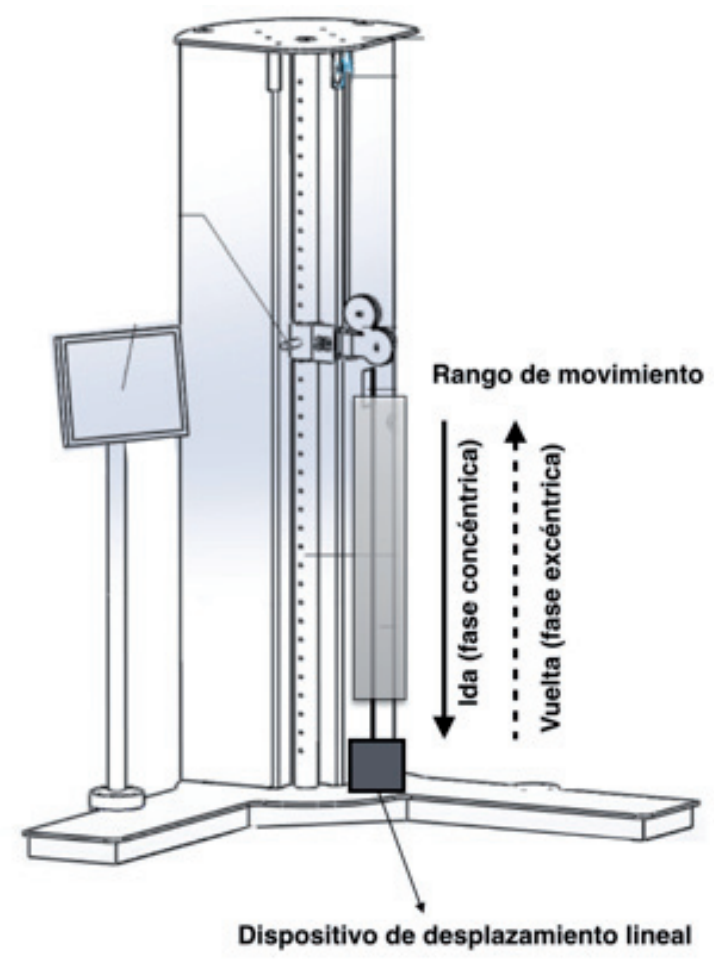

Análisis de la validez.- Para el análisis de la validez del dispositivo HHe 1.0 un DDL fue utilizado como "gold estándar”. La variable extraída del DDL fue la VRI. El protocolo realizado para testar la validación constaba de dos rangos de movimiento $(\mathrm{ROM})(0,25$ y $0,50 \mathrm{~cm})$, cuatro velocidades $\left(0,25,0,50,0,75 \mathrm{y} 1 \mathrm{~m} \cdot \mathrm{s}^{-1}\right)$, dos orientaciones (ida y vuelta) y dos cargas $(5$ y $15 \mathrm{~kg}$ ). Se realizaron un total de 20 repeticiones para cada una de las condiciones descritas.

Para determinar la fase isocinética se tuvo en cuenta el primer dato en el que se obtuvo la velocidad prefijada en cada una de las series realizadas con el dispositivo HHe 1.0. Además se registró el tiempo de inicio y el tiempo de fin (en ms) de la fase isocinética. Posteriormente, en función del tiempo de inicio y fin proporcionados por el dispositivo $\mathrm{HHe} 1.0$, se determinó la velocidad media en el rango isocinético en el DDL. En la Figura 2 se puede observar cómo se determinó la fase isocinética. Posteriormente se obtuvieron los datos de velocidad de cada una de las repeticiones realizadas tanto por el DDL como por el HHe 1.0.

Figura 2. Representación gráfica de una serie a $1.00 \mathrm{~m} \cdot \mathrm{s}^{-1}$, de $0,50 \mathrm{~cm}$ de rango de movimiento (ROM). La línea negra representa los valores de velocidad del dispositivo de desplazamiento lineal. La línea gris representa los valores de velocidad del Haefni Health System 1.0.

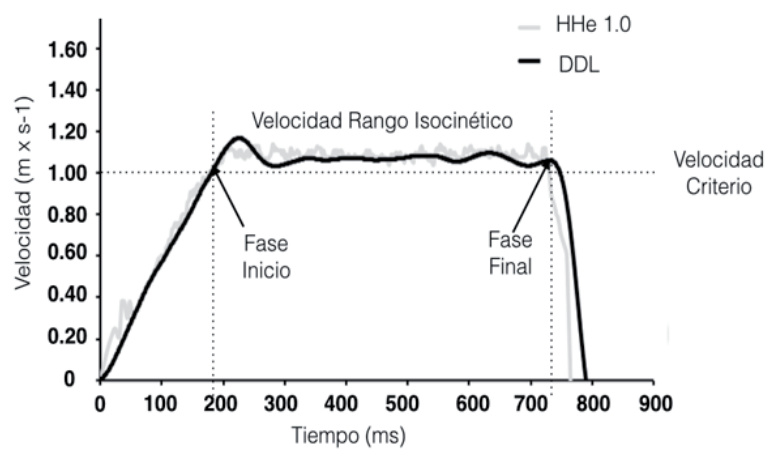

Análisis de la fiabilidad.- Un prueba test-retest fue realizada para el análisis de la fiabilidad del dispositivo HHe 1.0. El protocolo realizado fue: un total de 15 rangos de movimientos $(0,20 ; 0,22 ; 0,25 ; 0,27 ; 0,30 ; 0,32 ; 0,35 ; 0,37 ; 0,40$; $0,42 ; 0,45 ; 0,47 ; 0,50 ; 0,52 ; 0,55 \mathrm{~cm})$ con un total de 4 velocidades $\left(0,25,0,50,0,75 \mathrm{y} 1 \mathrm{~m} \cdot \mathrm{s}^{-1}\right)$, tanto para la orientación de ida (fase concéntrica) como para la orientación de vuelta (fase excéntrica), fueron evaluadas. Estas pruebas se repitieron en dos días diferentes (Día 1 y Día 2). Una repetición (ida y vuelta) se realizó por cada ROM evaluado. Se utilizó una carga de $5 \mathrm{~kg}$ para que se pudiera realizar los movimientos en el modo isocinético. La variable analizada fue la VRI.

\section{Material}

El material utilizado para los análisis de la validez y fiabilidad del la VRI del sistema HHe 1.0 fue un dispositivo de desplazamiento lineal (T-Force System, Ergotech, Murcia, España). Este dispositivo consta de un cable de extensión lineal 
en donde la velocidad es transmitida a un ordenador personal mediante una tarjeta de adquisición de datos analógico-digital de 14 bits de resolución. La velocidad instantánea vertical fue directamente tomada con una frecuencia de muestreo de $1000 \mathrm{~Hz}$. Se utilizó unas pesas calibradas de 5 y $15 \mathrm{~kg}$ marca Salter para realizar todas las evaluaciones. El sistema $\mathrm{HHe}$ 1.0 (iVolution R\&D', Granada, España) consta de un motor eléctrico de alta precisión con placa de conversión más una unidad de transmisión vía conexión IP. Se tuvieron en cuenta tanto la fase concéntrica como excéntrica del movimiento.

\section{Análisis estadístico}

Todas las variables fueron expresadas como media y desviación estándar (SD). El test de Kolmogorov-Smirnov fue utilizado para evaluar la normalidad de las variables analizadas. Para analizar las pruebas de validez se utilizó el Índice de Correlación Intraclase $\left(\mathrm{ICC}_{2,1}\right)$ y el coeficiente de correlación de Pearson. Para el análisis de la Fiabilidad se utilizó el ICC $_{2,1}$ además del Error Estándar de la Medida (SEM). La fórmula para el cálculo del SEM fue: . Donde MSe es la media cuadrática de los errores (Weir, 2005). Además se calculó para cada una de las condiciones de evaluación el coeficiente de variación $(\mathrm{CV})$, es decir, el valor de desviación estándar partido entre la media y multiplicado por 100. Para el análisis de la heterocedasticidad se computaron las diferencias de medias en relación a los valores individuales. Posteriormente, se procedió al cálculo del coeficiente de correlación para examinar formalmente la presencia de heterocedasticidad (Atkinson \& Neville, 1998). Un análisis bifactorial (día [2] x velocidad [4]) de la varianza de medidas repetidas (ANOVA MR) fue realizado para contrastar las medias de velocidad en los dos días de evaluación. Cuando no se cumplió el supuesto de esfericidad de Mauchly, la corrección de los grados de libertad fue realizada mediante la fórmula de Greenhouse-Geisser. El tamańo del efecto $\left(\eta^{2}\right)$ fue computado mediante eta cuadrado parcial. El nivel de significación estadística se estableció para $p<0,05$. Todas las pruebas fueron realizadas utilizando un paquete de análisis estadístico (SPSS, Inc, Illinois, EEUU).

\section{Resultados}

\section{Validez}

En la Tabla 2 y 3 se resumen los resultados de la media \pm SD de la VRI en todas las condiciones analizadas, para la ida (fase concéntrica) y la vuelta (fase excéntrica), respectivamente.

El ICC $_{2,1}$ obtenido para el análisis de la validez del protocolo en su conjunto, fue de 0,99 , tanto para la fase concéntrica (ida) como para la fase excéntrica (vuelta).

El análisis de la correlación de Pearson de las diferencias en valor absoluto y los valores individuales no mostraron tendencia lineal $\left(\mathrm{R}^{2}=0,008 ; \mathrm{p}>0,05\right)$. En la Figura 3 se presenta el gráfico de las diferencias (HHe 1.0 - DDL) de las pruebas del protocolo de validación. La media y desviación estándar de las diferencias fue de $-0,001 \pm 0,01 \mathrm{~m} \cdot \mathrm{s}^{-1}$.

Figura 3. Representación gráfica de las diferencias de velocidad (HHe 1.0 - DDL) en el rango isocinético de evaluación del protocolo de validez.

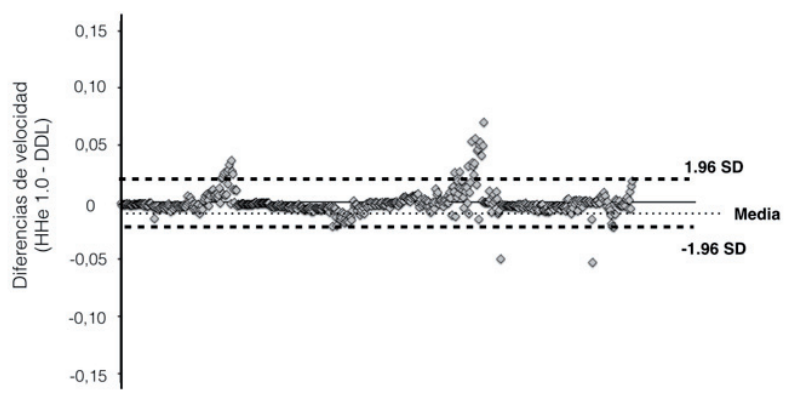

Tabla 1. Valores medios y desviación estándar de la velocidad en el rango isocinético en todo el protocolo realizado. Estos valores corresponde a la fase de ida (concéntrica).

\begin{tabular}{|c|c|c|c|c|c|c|c|c|}
\hline \multirow{3}{*}{$\begin{array}{l}\text { Carga }(\mathrm{Kg}) \\
\text { ROM }(\mathrm{cm}) \\
\text { Dispositivo }\end{array}$} & \multicolumn{4}{|c|}{5} & \multicolumn{4}{|c|}{15} \\
\hline & \multicolumn{2}{|c|}{0,25} & \multicolumn{2}{|c|}{0,50} & \multicolumn{2}{|c|}{0,25} & \multicolumn{2}{|c|}{0,50} \\
\hline & HHe 1.0 & $\mathrm{DDL}$ & HHe 1.0 & DDL & HHe 1.0 & DDL & HHe 1.0 & DDL \\
\hline Velocidad 1 & 0,25 & 0,25 & 0,25 & 0,25 & 0,25 & 0,25 & 0,25 & 0,24 \\
\hline$(0,25 \mathrm{~m} \times \mathrm{s}-1)$ & 0,001 & 0,001 & 0,001 & 0,001 & 0,001 & 0,001 & 0,001 & 0,004 \\
\hline Velocidad 2 & 0,49 & 0,49 & 0,49 & 0,49 & 0,50 & 0,50 & 0,50 & 0,50 \\
\hline$(0,50 \mathrm{~m} \times \mathrm{s}-1)$ & 0,001 & 0,001 & 0,001 & 0,001 & 0,001 & 0,002 & 0,001 & 0,002 \\
\hline Velocidad 3 & 0,75 & 0,74 & 0,75 & 0,74 & 0,76 & 0,76 & 0,75 & 0,75 \\
\hline$(0,75 \mathrm{~m} \times \mathrm{s}-1)$ & 0,003 & 0,003 & 0,004 & 0,001 & 0,002 & 0,004 & 0,001 & 0,006 \\
\hline Velocidad 4 & 1,05 & 1,04 & 1,05 & 1,04 & 1,05 & 1,04 & 1,04 & 1,03 \\
\hline$(1 \mathrm{~m} \times \mathrm{s}-1)$ & 0,001 & 0,001 & 0,001 & 0,003 & 0,001 & 0,001 & 0,002 & 0,011 \\
\hline
\end{tabular}

${ }^{*} \mathrm{HHe} 1.0$ = Haefni Health System 1.0 y DDL = dispositivo de desplazamiento lineal 
Tabla 2. Valores medios y desviación estándar de la velocidad en el rango isocinético en todo el protocolo realizado. Estos valores corresponde a la fase de vuelta (excéntrica).

\begin{tabular}{|c|c|c|c|c|c|c|c|c|}
\hline \multirow{3}{*}{$\begin{array}{c}\text { Carga }(\mathrm{Kg}) \\
\text { ROM }(\mathrm{cm}) \\
\text { Dispositivo }\end{array}$} & \multicolumn{4}{|c|}{5} & \multicolumn{4}{|c|}{15} \\
\hline & \multicolumn{2}{|c|}{0,25} & \multicolumn{2}{|c|}{0,50} & \multicolumn{2}{|c|}{0,25} & \multicolumn{2}{|c|}{0,50} \\
\hline & HHe 1.0 & DDL & HHe 1.0 & DDL & HHe 1.0 & DDL & HНe 1.0 & DDI \\
\hline Velocidad 1 & 0,25 & 0,25 & 0,25 & 0,25 & 0,25 & 0,25 & 0,25 & 0,24 \\
\hline$(0,25 \mathrm{~m} \mathrm{x} \mathrm{s}-1)$ & 0 & 0,001 & 0,003 & 0,003 & 0,003 & 0,001 & 0,001 & 0,001 \\
\hline Velocidad 2 & 0,50 & 0,50 & 0,49 & 0,47 & 0,49 & 0,49 & 0,48 & 0,47 \\
\hline$(0,50 \mathrm{~m} \times \mathrm{s}-1)$ & 0,002 & 0,002 & 0,001 & 0,002 & 0,001 & 0,003 & 0,001 & 0,001 \\
\hline Velocidad 3 & 0,75 & 0,76 & 0,75 & 0,74 & 0,76 & 0,75 & 0,75 & 0,75 \\
\hline$(0,75 \mathrm{~m} \times \mathrm{s}-1)$ & 0,002 & 0,004 & 0,001 & 0,004 & 0,003 & 0,011 & 0,001 & 0,01 \\
\hline Velocidad 4 & 1,04 & 1,03 & 1,04 & 1,03 & 1,04 & 1,04 & 1,04 & 1,03 \\
\hline$(1 \mathrm{~m} \times \mathrm{s}-1)$ & 0,002 & 0,003 & 0,005 & 0,004 & 0,002 & 0,001 & 0,002 & 0,011 \\
\hline
\end{tabular}

${ }^{*} \mathrm{HHe} 1.0$ = Haefni Health System 1.0 y DDL = dispositivo de desplazamiento 1

Fiabilidad

En la Tabla 3 se muestran los valores medios \pm SD de las velocidades en función de la velocidad criterio evaluada y de las diferencias de velocidad.

Tabla 3. Valores medios y desviación estándar de la velocidad en el rango isocinético en todo el protocolo de fiabilidad realizado.

\begin{tabular}{|c|c|c|c|c|}
\hline Fase del movimiento & $\begin{array}{c}\text { Velocidad Criterio } \\
(\mathrm{m} \times \mathrm{s}-1)\end{array}$ & $\begin{array}{c}\text { Velocidad Día } 1 \\
(\mathrm{~m} \times \mathrm{s}-1)\end{array}$ & $\begin{array}{c}\text { Velocidad Día } 2 \\
(\mathrm{~m} \times \mathrm{s}-1)\end{array}$ & $\begin{array}{l}\text { Diferencia Velocidad (D2-D1) } \\
(\mathrm{m} \times \mathrm{s}-1)\end{array}$ \\
\hline \multirow{4}{*}{ Ida (concéntrico) } & 0,25 & $0,257(0,0005)$ & $0,258(0,0005)$ & $0,0003(0,0005)$ \\
\hline & 0,50 & $0,502(0,003)$ & $0,502(0,003)$ & $0,0001(0,0021)$ \\
\hline & 0,75 & $0,753(0,013)$ & $0,756(0,012)$ & $0,0019(0,0032)$ \\
\hline & 1 & $1,049(0,047)$ & $1,05(0,046)$ & $0,0015(0,0024)$ \\
\hline \multirow{4}{*}{ Vuelta (excéntrico) } & 0,25 & $0,251(0,0007)$ & $0,251(0,0008)$ & $0,0003(0,0005)$ \\
\hline & 0,50 & $0,505(0,003)$ & $0,506(0,002)$ & $0,0001(0,0021)$ \\
\hline & 0,75 & $0,752(0,006)$ & $0,752(0,007)$ & $0,0005(0,0032)$ \\
\hline & 1 & $1,045(0,006)$ & $1,048(0,023)$ & $0,0027(0,0024)$ \\
\hline
\end{tabular}

La prueba test-retest realizada para la variable de la VRI mostró un $\mathrm{ICC}_{2,1}$ de $0,99\left(0.99-1 ; 95 \% \mathrm{IC} \mathrm{ICC}_{2,1}\right)$, analizando todas la condiciones en su conjunto. La correlación de Pearson de la VRI en ambos días de evaluación fue de $\mathrm{r}=$
$0,999$ ( $\mathrm{p}<0,001)$, tanto para la orientación de ida (fase concéntrica) como para la orientación de vuelta (fase excéntrica). La Tabla 4 muestra los estadísticos de fiabilidad para cada una de las condiciones evaluadas.

Tabla 4. Estadísticos de fiabilidad (Media, SD, SEM, $\mathrm{ICC}_{2,1}$ y CV) de la velocidad en el rango isocinético en todo el protocolo de fiabilidad realizado.

\begin{tabular}{|c|c|c|c|c|c|}
\hline Fase del movimiento & Velocidad Criterio $(\mathrm{m} \times \mathrm{s}-1)$ & Velocidad Media (SD) & SEM (m x s-1) & ICC (95\%IC) & $\mathrm{CV}(\%)$ \\
\hline \multirow{4}{*}{ Ida (concéntrico) } & 0,25 & $0,257(0,0005)$ & 0,0001 & $0.71(0.13-0.90)$ & 0,19 \\
\hline & 0,50 & $0,502(0,003)$ & 0,0001 & $0.84(0.52-0.95)$ & 0,60 \\
\hline & 0,75 & $0,755(0,012)$ & 0,0017 & $0.99(0.94-0.99)$ & 1,59 \\
\hline & 1 & $1,050(0,046)$ & 0,0017 & $0.99(0.99-1)$ & 4,38 \\
\hline \multirow{4}{*}{ Vuelta (excéntrico) } & 0,25 & $0,251(0,0004)$ & 0,0001 & $0.90(0.71-0.97)$ & 0,16 \\
\hline & 0,50 & $0,508(0,004)$ & 0,0017 & $0.93(0.79-0.98)$ & 0,79 \\
\hline & 0,75 & $0,752(0,006)$ & 0,0017 & $0.97(0.91-0.99)$ & 0,80 \\
\hline & 1 & $1,046(0,039)$ & 0,0042 & $0.98(0.95-0.99)$ & 3,73 \\
\hline
\end{tabular}


La Figura 4 representa el gráfico de las diferencias de la VRI (D2-D1; fase concéntrica [a] y fase excéntrica [b]). Los límites de acuerdo fueron $0,0011 \pm 0,0018 \mathrm{~m} \cdot \mathrm{s}^{-1}$ y $0,0011 \pm$
$0,0065 \mathrm{~m} \cdot \mathrm{s}^{-1}$, para la fase concéntrica y excéntrica, respectivamente.

Figura 4. Representación gráfica de las diferencias de velocidad $\left(\mathrm{m} \cdot \mathrm{s}^{-1}\right)$ en el rango isocinético de evaluación del protocolo de fiabilidad. [A] Diferencias de velocidad ida (fase concéntrica) [B] Diferencias de velocidad vuelta (fase excéntrica).

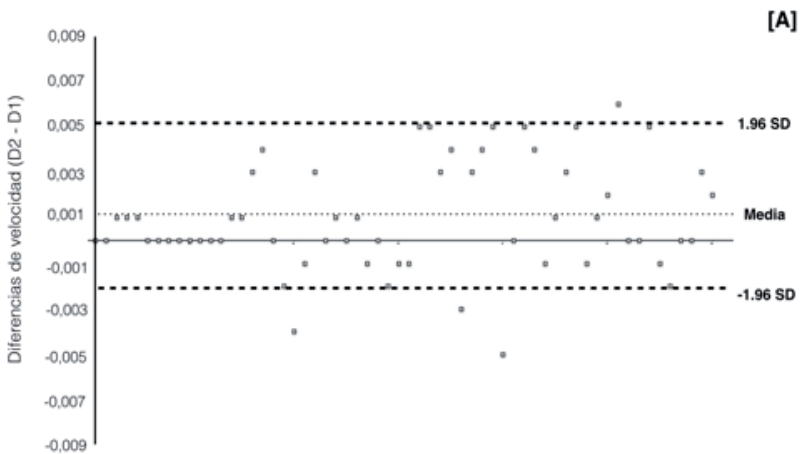

El ANOVA MR no mostró diferencias significativas $\left(\mathrm{F}_{[3,}\right.$ $\left.{ }_{42}=2,23 ; \mathrm{p}=0,089 ; \eta^{2}=0.142\right)$ en la interacción día $\mathrm{x}$ velocidad para la fase de ida. Del mismo modo, no se encontraron diferencias significativas $\left(\mathrm{F}_{[1.23,17.25]}=1,712 ; \mathrm{p}=0,179 ; \eta^{2}\right.$ $=0,109)$ en la interacción día velocidad para la fase de vuelta.

El análisis de la correlación de Pearson las diferencias en valor absoluto y lo valores individuales no mostró tendencia lineal $\left(\mathrm{R}^{2}=0,16 ; \mathrm{p}>0,05\right.$ y $\left.\mathrm{R}^{2}=0,13 ; \mathrm{p}>0,05\right)$, tanto para la fase concéntrica como para la fase excéntrica, respectivamente. Por lo tanto, los datos fueron homocedásticos.

\section{Discusión}

El objetivo principal de este trabajo fue analizar la validez y fiabilidad del dispositivo HHe 1.0 para la medición de la velocidad en el rango isocinético. Para ello, diferentes protocolos de evaluación fueron diseñados. En cuanto a las pruebas de validez se refieren, el ICC $_{2,1}$ obtenido en la variable de VRI fue de 0,998. Como se puede apreciar en las Tablas 1 y 2 los estadísticos descriptivos, independientemente de la fase concéntrica o excéntrica (ida o vuelta), el comportamiento de las medias y desviaciones de la VRI fueron similares en ambos dispositivos de evaluación (DDL como gold estándar y HHe 1.0 como dispositivo a evaluar). Esta misma comparativa se puede comprobar en la Figura 3. La media de las diferencias de velocidad entre ambos dispositivos fue de $-0,001 \pm 0.01$ $\mathrm{m} \cdot \mathrm{s}^{-1}$. A modo de ejemplo, en la Figura 4 se representa una repetición isocinética representativa a la velocidad de $1.00 \mathrm{~m} \cdot \mathrm{s}^{-}$ ${ }^{1}$. Teniendo en cuenta que el DDL proporciona una medida de velocidad y comparando los resultados de dichas medidas con las obtenidas en el dispositivo HHe 1.0, es consecuente decir que el dispositivo HHe 1.0 es capaz de medir veloci-

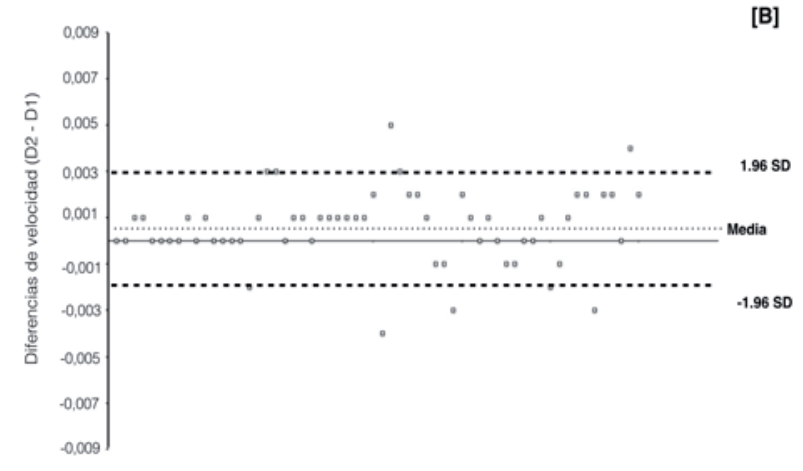

dad. Por lo tanto, el concepto de validez está asegurado. En la bibliografía es posible encontrar diferentes trabajos que analizan la validez y fiabilidad mecánica de dispositivos isocinéticos (Drouin et al., 2004; Orri y Darden, 2008). El trabajo realizado por Drouin et al. (2004), analizaron la validez para la medición de la velocidad en el dispositivo Biodex System 3 Pro, encontrando valores muy altos de $\mathrm{ICC}_{2.1}$ (rango 0,99-1). Sin embargo, a diferencia de este y otros trabajos en donde validación sólo se pudo testar durante la fase concéntrica. En nuestro estudio ambas fases del movimiento fueron analizadas obteniendo resultados muy prometedores. Esto nos asegura una adecuada medición de la velocidad en el rango isocinético en ambas fases del movimiento.

En cuanto a las pruebas de fiabilidad se refieren, los resultados mostraron una excelente fiabilidad parcial $\left(\mathrm{ICC}_{2,1}=\right.$ $0,99)$ para la VRI en las pruebas test-retest realizadas, tanto para la fase concéntrica como para la fase excéntrica. Este resultado fue obtenido cuando se analizaron todas las cargas en su conjunto. Sin embargo, también se analizó la fiabilidad para cada una de las condiciones de velocidad criterio evaluadas $\left(0,25,0.50,0,75\right.$ y $\left.1,00 \mathrm{~m} \cdot \mathrm{s}^{-1}\right)$. En la Tabla 4 se resumen los valores de la media, SD, SEM, ICC , $_{2,1}$ CV para cada una de las condiciones de VRI analizadas. Además de aportar un resultado global de la fiabilidad $\left(\mathrm{ICC}_{2,1}=0,99\right)$ se optó por proporcionar un resultado por cada una de las velocidades criterio. Desde el conocimiento de los autores del presente trabajo, entendemos que este dispositivo (HHe 1.0) se va a utilizar en una condición en concreto para cada vez. Esto es, que cuando se realice ejercicio isocinético, se realizará con un ROM y una velocidad determinada y no todas al mismo tiempo. En la Tabla 4 se aprecia como las diferencias de velocidad entre los dos días de evaluación fueron muy bajas. El ANO- 
VA MR realizado no mostró diferencias significativas en la interacción día $\mathrm{x}$ intensidad. Esto nos podría estar indicando dos cosas. En primer lugar, que independientemente del día de evaluación, la respuesta del dispositivo es estable, es decir, es fiable. En segundo lugar, que el error sistemático es escaso o nulo (Atkinson \& Neville, 1998). Sin embargo, resulta primordial recalcar que los ICC $_{2.1}$ por cada velocidad analizada iban aumentando conforme aumenta la velocidad criterio. Es importante señalar que para una adecuada interpretación de los resultados, debido a la baja variabilidad encontrada en las series a velocidad lenta (p.e. 0,25 $\mathrm{m} \cdot \mathrm{s}^{-1}$ ), los indicadores de fiabilidad parcial $\left(\mathrm{ICC}_{2.1}\right.$ ) fueron bajos (ver Tabla 4). Pero cuando recurrimos a los estadísticos de fiabilidad absoluta (SEM y CV), es posible percibir que en las series donde la velocidad fue más lenta estos estadísticos fueron más bajos en comparación con las velocidad más altas (ver Tabla 4). Este problema fue descrito por Weir (2005). El mencionado autor recomienda basarse además del ICC en otros estadísticos para una adecuada interpretación de los resultados.

Otros estudios (Comstock, et al., 2011; Feiring, Ellenbecker, y Derscheid, 1990; Gómez-Piriz, Sánchez, Manrique, y González, 2012; Maffiuletti, Bizzini, Desbrosses, Babault, y Munzinger, 2007; Requena et al., 2012) han analizado la fiabilidad de diferentes dispositivos de evaluación utilizando una muestra compuesta por personas. El principal problema a la hora de utilizar personas en la validación de un instrumento de evaluación es la variabilidad biológica introducida por el propio sujeto (Hopkins, 2000; Cejudo, Baranda, Ayala, y Santonja, 2012). En nuestro estudio se han realizado las pruebas de validez y de fiabilidad sin introducir este error biológico, puesto que las repeticiones fueron realizadas por el motor del dispositivo HHe 1.0. Por lo tanto, el error biológico introducido por los sujetos quedó completamente eliminado. La principal dificultad que tuvimos durante el procedimiento de evaluación con el dispositivo utilizado como gold estándar, es decir, con el DDL. Durante las evaluaciones con velocidades lentas no existió ningún contratiempo puesto que el desplazamiento de la pesa y del cable del DDL fue mínimo, por eso se obtuvieron valores $\mathrm{CV}$ menores del $1 \%$. En cambio, cuando la velocidad criterio aumentó i.e. de 0,25 a $0,75 \mathrm{~m} \cdot \mathrm{s}^{-1}$, se puede apreciar en la Figura 4, como las diferencias en la variable de VRI aumentaron. En la Tabla 4 se puede observar que a pesar del alto valor del $\mathrm{ICC}_{2,1}(0,99)$, en las velocidades más altas (i.e. $1 \mathrm{~m} \cdot \mathrm{s}^{-1}$ ), el $\mathrm{CV}$ fue de 4,38 y 3,73\% para la fase de ida y vuelta, respectivamente. Aun con todos estos inconvenientes, los valores de CV fueron aceptables. Drouin et al. (2004) analizaron la validez y fiabilidad mecánica del dispositivo isocinético Biodex System 3 Pro para la medición de la velocidad isocinética, posición y torque. Estos investigadores analizaron la fiabilidad de las variables proporcionadas por el dispositivo mediante pruebas "trial-to-trial" y "day-today". Un investigador aceleraba manualmente el dispositivo y el ROM a testar fue de $70^{\circ}$. Los resultados fueron altos, rango ICC entre 0,99 y 1 , tanto para las medidas trial-totrial como para las de day-to-day. En cuanto a la validez del dispositivo los resultados mostraron bajos CV (inferiores al $12 \%$ ). El principal problema que encontraron fue durante las velocidades más altas (a partir de 300 o/sec). La velocidad criterio fue mayor que la velocidad real. Por lo tanto, los autores argumentaron que las medidas obtenidas fueron fiables y válidas hasta llegar a dicha velocidad angular. Por su parte Orri y Darden (2008) analizaron la validez y fiabilidad de un nuevo dispositivo isocinético llamado iSAM 9000. Como "gold estándar" para analizar la validez y fiabilidad utilizaron otro dispositivo isocinético Cybex 6000. Diferentes ejercicios fueron testados i.e. flexión/extensión de rodilla, hombros y tronco. Un total de 60 sujetos formaron parte de dicha investigación. Altos coeficientes de correlación de Pearson y de ICC, además de bajos-moderados SEM permitieron a los autores concluir con que el dispositivo analizado poseía una alta precisión y reproducibilidad. Aunque como se ha comentado anteriormente, mediante este tipo de pruebas no es posible conocer que parte del error total es asociado al dispositivo de evaluación y que parte es asociado a la variabilidad biológica del sujeto evaluado (Bruton, Conway, y Holgate, 2000).

Una de las principales limitaciones de este estudio fue que durante las evaluaciones realizadas para medir la VRI, conforme aumentaba la velocidad en el dispositivo HHe 1.0, se producía un rebote de las pesas al inicio y al final del movimiento. Como consecuencia, el cable del DDL utilizado para evaluar la variable de la velocidad sufría ligeros movimientos laterales, introduciendo una variabilidad en los datos obtenidos. Futuras investigaciones son necesarias utilizando otro tipo de dispositivo como medida gold estándar como por ejemplo, una cámara de alta velocidad y la inclusión de unas bandas elásticas para minimizar el rebote producido por la pesa. Sin embargo, como el objetivo del presente trabajo fue analizar la validez y fiabilidad del nuevo dispositivo HHe 1.0 en la medición de la VRI, estas variaciones tuvieron poca influencia sobre los resultados de la validez y fiabilidad ya que tanto la fase de aceleración como la fase de frenado fueron excluida de los análisis.

\section{Conclusiones}

Las velocidades obtenidas en el modo isocinético por el sistema HHe 1.0 fueron válidas y fiables Por lo tanto, los resultados obtenidos en esta investigación avalan la validez y fiabilidad del dispositivo HHe 1.0 para la medición de la velocidad en el rango isocinético.

\section{Conflicto de intereses}

Los autores no tienen interés comercial o conflicto de intereses sobre este dispositivo. 


\section{Referencias}

1. Atkinson, G., y Neville, A. M. (1998). Statistical Methods For Assessing Measurement Error (Reliability) in Variables Relevant to Sports Medicine. Sports Medicine, 25(8): 217 - 238.

2. Bruton, A., Conway, J. H., y Holgate, S. T. (2000). 'Reliability: What is it and how it is measured?'. Physiotherapy, 86(2): $94-95$.

3. Cejudo, A., Baranda, P. S. de, Ayala, F., y Santonja, F. (2012). Fiabilidad absoluta de 2 pruebas de valoración del rango de movimiento del tobillo en jugadores de balonmano. Cuadernos de Psicología del Deporte, 12(2), 23-29.

4. Comstock, Solomon-Hill., G., Flanagan, S. D., Earp, J. E., Luk, H. Y., Dorbins, K. A., Dunn-Lewis, C., Fragala, M. S., Ho, J. Y., Hatfield, D. L., Vingren, J. L., Denegar, C. R., Volek, J. S., Kupchak, B. R., Maresh, C. M., y Kraemer, W. J. (2011). Validity of the Myotest in measuring force and power production in the Squat and Bench Press. The Journal of Strength and Conditioning Research, 27(6): 2293 - 2297.

5. Drouin, J. M., Valovich-mcLeod, T. C., Shultz, S. J., Gansneder, B. M., y Perrin D. H. (2004). Reliability and validity of the Biodex system 3 pro isokinetic dynamometer velocity, torque and position measurements. European Journal of Applied Physiology, 91: 22 - 29.

6. Feiring, D. C., Ellenbecker, T. S., y Derscheid, G. L. (1990). Test-retest Reliability of the biodex isokinetic dynamometer. Journal of Orthopedics and Sport Physical Therapy, 11(7): 298-300.

7. Gomez-Piriz, P. T., Sanchez, E. T., Manrique, D. C., y Gonzalez, E. P. (2012). Reliability and Comparability of the Accelerometer and the Linear Position Measuring Device in Resistance Training. The Journal of Strength and Conditioning Research, 27(6): 1664 - 1670.

8. Hopkins, W. G. (2000). Measures of reliability in sports medicine and science. Sports Med. 30: 375-381.

9. Maffiuletti, N. A., Bizzini, M., Desbrosses, K., Babault, N, y Mun- zinger, Urs. (2007). Relialibity of knee extensión and flexion measurements using the Con-Trex isokinetic dynamometer. Clinical Physiologya and Functional Imaging, 27: $346-353$.

10. Orri, J. C., y Darden, C. F. (2008). Technical report: reliability and validity of the isam 9000 isokinetic dynamometer. Journal of Strength and Conditioning Research, 22(1): 310 - 317.

11. Patterson, L. A., y Spivey, W. E. (1992). Validity and Reliabiliy of the LIDO Active isokinetic System, Journal of Orthopedics and Sport Physical Therapy 15(1): $32-36$

12. Requena, B., Requena, F., García, I., Saez-Saez de Villarreal, E., y Pääsuke, M. (2012). Reliability and validity of a wireless microelectromechanicals based system (Keimove ${ }^{\mathrm{TM}}$ ) for measuring vertical jumping performance. Journal of Sports Science and Medicine, (11): 115 - 122.

13. Sole, G., Hamrén, J., Milosavljevic, S., Nicholson, H., y Sullivan, S J. (2007). Test-Retest Reliability of Isokinetic Knee Extension and Flexion. Archives of Physical Medicine and Rehabilitation, 88(5), 626631.

14. Toonstra, J., y Mattacola, C. G. (2012). Test-retest Reliability and Validity of Isometric Knee Flexion and Extension Measurement Using Three Methods of Assessing Muscle Strength. Journal of Sport Rehabilitation, 83(6): $811-815$.

15. Tsiros, M. D., Grimshaw, P. N., Shield, A. J., y Buckley, J. D. (2011). Test - Retest Reliability of the Biodex System 4 Isokinetic Dynamometer fo Knee Strength Assessment in Paediatric Populations. Journal of Allied Heath, 40(3): 115-119.

16. Weir, J. P. (2005). Quantifying Test-Retest Reliability Using the Intraclass Correlation Coefficient and The SEM. Journal of Strength and Conditioning Research, 19(1): $231-240$. 